\title{
El juego como estrategia didáctica para el fortalecimiento de las competencias ciudadanas en la construcción de la paz, en los estudiantes de quinto grado de básica primaria, a través del juego popular denominado la vuelta a Colombia.
}

\author{
The game as a didactic strategy for the strengthening of citizens 'competences in the \\ construction of peace, in the students of fifth grade of primary basics, through the people's \\ game denominated the return to Colombia. \\ Juan Carlos Pérez-Velásquez ${ }^{\mathrm{a}^{*}}$, Liliana Esperanza Peñaranda-Mojica ${ }^{\mathrm{b}}$ \\ a* Magister en Educación, juancperez1720@gmail.com, orcid.org/0000-0001-8459-2047, Colegio Santos Apóstoles, Cúcuta, Colombia
bMagister en Educación, lilianita3181@hotmail.com, orcid.org/0000-0001-8459-2047, Colegio Santos Apóstoles, Cúcuta, Colombia
}

Forma de citar: Pérez-Velásquez, J.C, Peñaranda-Mojica. L.E El juego como estrategia didáctica para el fortalecimiento de las competencias ciudadanas en la construcción de la paz, en los estudiantes de quinto grado de básica primaria, a través del juego popular denominado la vuelta a Colombia.. Eco Matemático, 10

(2), 47-53

Recibido: 22 febrero 2019

Aceptado: 30 abril 2019

\section{Palabras clave \\ Competencias \\ ciudadanas, convivencia, autoestima, tolerancia, paz}

*Autor para correspondencia:djuancperez1720@gmail.com los rodean.
Resumen:El proyecto estuvo basado en el fortalecimiento de las competencias ciudadanas de los estudiantes de una Institución Educativa de Cúcuta, Norte de Santander, en los cuales, por medio de un diagnóstico, se pudo detectar graves falencias en dicho aspectos, y más específicamente en aspectos de convivencia, tolerancia y respeto por la opinión, creencias y opiniones de las personas que

Se diseñó y aplico diversas actividades conducentes a mejorar dichos aspectos, de lo cual se debe mencionar que fueron altamente efectivas, dadas las estrategias aplicadas, en las cuales se tuvo en cuenta la autoestima, la reflexión y la visualización de los propios defectos y virtudes de cada estudiante, se planteó una observación directa, con enfoque cualitativo que permitió establecer que los estudiantes, entre otras cosas, no se apropian de los problemas de su entorno y se les dificulta reconocer sus falencias en lo referente a la convivencia y al respeto por las ideas y creencias de los demás. Los instrumentos que se utilizaron para la recolección de la información fueron el diario pedagógico y rejilla diagnostica. A través del juego denominado "la Vuelta a Colombia" se observó que se fortalecieron las competencias ciudadanas en los estudiantes de quinto grado donde ellos proponen soluciones prontas y afectivas.

Los resultados fueron socializados a la comunidad educativa y el proyecto se dejó inmerso en un plan de mejoramiento institucional el cual será implementado en todas las áreas, cursos y sedes de la Institución Educativa. 


\section{Keywords}

Citizenship skills, coexistence, self-esteem, tolerance, peace

\section{Introducción}

La investigación tuvo como objetivo la implementación del juego como estrategia para el fortalecimiento de las competencias ciudadanas de los estudiantes de las instituciones educativas de Norte de Santander, con el ánimo de mejorar la relación entre estudiantes y entre estos y sus docentes, así como con su contexto social y familiar.

Se plantearon una serie de actividades donde los estudiantes se empezaron a reconocer como elementos constitutivos de una sociedad, que, en medio de un proceso de paz, exige de su compromiso y aporte a la convivencia y a la tolerancia, aspectos de los que en la actualidad adolece la sociedad actual.
Una vez planteadas las actividades, se procedió a su implementación y desarrollo, lo cual al principio resulto un tanto difícil, pues es evidente que a las personas se les dificulta reconocer sus errores y reconocer las virtudes y derechos de los demás. Sin embargo, en la medida en que se avanzaba en el proyecto se fueron disipando dichas actitudes y se fueron logrando los objetivos propuestos.

Al finalizar el desarrollo de las actividades, se logró percibir un positivo cambio de actitud de los estudiantes, reconociendo sus falencias y a la vez valorando, la opinión, creencia y palabra de las personas que lo rodean, así como la apropiación de las problemáticas sociales como propias, sugiriendo de esta manera correctivos y soluciones claras y concretas. 
Metodología

Esta propuesta de estrategias pedagógicas para fortalecer las competencias ciudadanas en los estudiantes del quinto grado de básica primaria del Colegio Los Santos Apóstoles, se llevará a cabo a través de un enfoque investigativo cualitativo, el cual según (Hernández Sampieri, FernándezCollado, \& Baptista, 2004), utiliza la recolección de datos sin medición numérica para descubrir o afinar preguntas de investigación en el proceso de interpretación

De acuerdo con (Todd, Nerlich y McKeown, 2004), citados por (Hernández Sampieri, FernándezCollado, \& Baptista, 2004), el enfoque cualitativo se basa en métodos de recolección de datos no estandarizados. No se efectúa una medición numérica, por lo cual el análisis no es estadístico. La recolección de los datos consiste en obtener las perspectivas y puntos de vista de los participantes (sus emociones, experiencias, significados y otros aspectos subjetivos).

También resultan de interés las interacciones entre individuos, grupos y colectividades. El investigador pregunta cuestiones generales $\mathrm{y}$ abiertas, recaba datos expresados a través del lenguaje escrito, verbal y no verbal, así como visual, los cuales describe y analiza y los convierte en temas, esto es, conduce la indagación de una manera subjetiva y reconoce sus tendencias personales.

\section{Instrumentos para la recolección de la información}

La recolección de la información requiere el diseño de instrumentos que permitan dar cuenta de los resultados de forma objetiva y coherente con el planteamiento de los objetivos de la investigación. En tal sentido se proponen los siguientes:

Instrumento $\mathbf{N}^{\mathbf{0}}$ 1. Diario Pedagógico para el registro de las actividades y observaciones realizadas.
Instrumento $\mathbf{N}^{\mathbf{0}}$ 2. Rejilla diagnóstica con el fin de observar el comportamiento de los estudiantes desde la perspectiva de competencias ciudadanas.

\section{La vuelta a Colombia}

Población: Niños, niñas, adolescentes y adultos

Clasificación: Juego de competencia; juego de circuito; juego de estrategia.

Edad: 7 años en adelante

Habilidades y/o capacidades a desarrollar: motricidad fina, pensamiento estratégico, pensamiento espacial, trabajo en equipo.

Materiales: Canicas, diversos elementos que funcionen como obstáculos (piedras, ramas, tubos, etc.).

Campo de juego: relativamente amplío; circuito-pista con diversos obstáculos, por lo general en forma del mapa de Colombia.

Reglas y/o descripción: El objetivo principal de este juego es recorrer una zona demarcada previamente en el suelo (por lo general, el mapa de Colombia), sin embargo en esta se encontraran ciertas trampas (agujeros, túneles, etc.). Este recorrido debe realizarse con bolitas de cristal o canicas.

Para dar inicio al juego se rifa por parte del observador de base el equipo que inicia, el ganador golpea la bolita con la uña del dedo índice tratando de recorrer la mayor distancia y debe esperar que el último participante termine para tener derecho al siguiente turno, debe ser alterno entre equipos, gana quién que logre llegar a la meta en los 10 minutos. (Aunque existe diversa modalidades). 


\section{Resultados}

\section{Rejilla diagnóstica}

El diagnóstico para el presente proyecto se realizó por medio de la observación directa no participante, el cual está basado en las competencias ciudadanas para quinto grado; de lo que se debe rescatar los siguientes aspectos:

Los estudiantes en mención no poseen la capacidad de reflexionar sobre problemáticas sociales de su entorno social, académico y escolar, aunque no desconocen la existencia de los mismos dentro de su contexto.

En ese mismo sentido los estudiantes no participan en la búsqueda de soluciones a problemas sociales, pues no se sienten parte de ellos, ni mucho menos de su solución.

Los estudiantes, en su mayoría no tienen en cuenta los puntos de vista ajenos, generando de esta manera inconformidad y malas relaciones interpersonales.

Los estudiantes, en su mayoría, no participan en la búsqueda de soluciones a problemas sociales y del bienestar de su comunidad, denotando apatía y negligencia ante ellos.

Por último, no comprende que los problemas y sus soluciones involucran distintas dimensiones ni reconoce relaciones entre éstas.

\section{Rejilla diagnóstica}

El diagnóstico para el presente proyecto se realizó por medio de la observación directa no participante, el cual está basado en las competencias ciudadanas para quinto grado; de lo que se debe rescatar los siguientes aspectos:

Los estudiantes en mención no poseen la capacidad de reflexionar sobre problemáticas sociales de su entorno social, académico y escolar, aunque no desconocen la existencia de los mismos dentro de su contexto.

En ese mismo sentido los estudiantes no participan en la búsqueda de soluciones a problemas sociales, pues no se sienten parte de ellos, ni mucho menos de su solución.

Los estudiantes, en su mayoría no tienen en cuenta los puntos de vista ajenos, generando de esta manera inconformidad y malas relaciones interpersonales.

Los estudiantes, en su mayoría, no participan en la búsqueda de soluciones a problemas sociales y del bienestar de su comunidad, denotando apatía y negligencia ante ellos.

Por último, no comprende que los problemas y sus soluciones involucran distintas dimensiones ni reconoce relaciones entre éstas.

\section{Conclusiones}

Teniendo en cuenta que las conclusiones se plantean a partir de los objetivos propuestos en el proyecto, se relacionan a continuación, sus generalidades.

Como primer punto se debe decir que se fortalecieron las competencias ciudadanas para la construcción de la paz, a través del juego popular denominado "La vuelta a Colombia" en los estudiantes del grado quinto de primaria de la Institución Educativa Santos Apóstoles de la ciudad de Cúcuta, lo cual se logró mediante una metodología previamente establecida y ordenada, por medio de la cual se dio pleno cumplimiento a los objetivos específicos así:

Se determinaron algunos aspectos que afectan la convivencia en los estudiantes de quinto grado de la Institución Educativa Los Santos Apóstoles de la ciudad de Cúcuta, de los cuales se pueden destacar los siguientes aspectos: 


\section{Los estudiantes:}

No poseen la capacidad de reflexionar sobre problemáticas sociales de su entorno social, académico y escolar

No participan en la búsqueda de soluciones a problemas sociales. No tienen en cuenta los puntos de vista ajenos, generando inconformidad y malas relaciones interpersonales.

No participan en la búsqueda de soluciones a problemas sociales y del bienestar de su comunidad.

No comprenden que los problemas y sus soluciones involucran distintas dimensiones ni reconoce relaciones entre éstas.

De acuerdo con lo anterior, se diseñaron estrategias didácticas basadas en el juego popular denominado "La vuelta a Colombia por la paz" basadas en la construcción de paz en el posconflicto.

De lo anterior se debe destacar que en cada una de las actividades se tuvieron en cuenta las competencias básicas que deben desarrollar los estudiantes en lo referente a la convivencia, la tolerancia y el respeto.

Una vez diseñadas las actividades, se procedió a su aplicación con los estudiantes en mención, para luego hacer una evaluación cualitativa del proceso, de lo cual se pueden destacar los siguientes aspectos:

\section{Los estudiantes:}

Mejoraron de forma significativa la capacidad de reflexionar sobre problemáticas sociales de su entorno social, académico y escolar.

Participan en la búsqueda de soluciones a problemas sociales
Tienen en cuenta los puntos de vista ajenos, lo cual genera mayor confianza con sus compañeros y mejora las relaciones sociales y familiares.

Participan en la búsqueda de soluciones a problemáticas sociales y del bienestar de su comunidad.

Comprenden que los problemas y sus soluciones involucran distintas dimensiones y reconocen relaciones entre éstas.

En cuanto a la triangulación efectuada en el proyecto, se pueden destacar los siguientes aspectos:

Cuando los estudiantes reconocen las competencias ciudadanas como algo más que una teoría, dimensionan los problemas que se dan al interior de las aulas y de esta manera proponen soluciones prontas y efectivas, lo cual guarda plena concordancia con lo expuesto por (Ruiz \& Chaux, 2005) al referirse a la competencia ciudadana como "el conjunto de capacidades y habilidades cognitivas, emocionales y comunicativas -integradas- relacionadas con conocimientos básicos (contenidos, procedimientos, mecanismos) que orientan moral y políticamente nuestra acción ciudadana".

Por otra parte, se puede mencionar que la función del entorno familiar en lo referente a los valores es de gran importancia en todos los ámbitos sociales en que se mueve el estudiante y que todos los valores e incluso los antivalores que trae el estudiante de su casa se evidencian en su comportamiento en el aula, lo cual debe ser ajustado por los docentes y compañeros de acuerdo con cada situación específica lo cual se conjuga de manera directa con lo expuesto por (Arufe-Giráldez, 2011), quien afirma que los valores se constituyen y se aprenden fundamentalmente en la infancia y la adolescencia, a partir de los modelos sociales de padres/madres, docentes, medios de comunicación 
y amigos/as. De ahí la gran importancia de la intervención del docente.

\section{Referencias}

Altablero. (Marxo de 2004). Competencias ciudadanas Habilidades para saber vivir en paz. Recuperado el 29 de Enero de 2017, de http:// www.mineducacion.gov.co/1621/article-87283. html

Arufe-Giráldez, V. (2011). La educación en valores en el aula de educación. ¿Mito o realidad? Revista digital de educación física(9), 32-42.

Bausela, E. (2001). La docencia a traves de la investigación-acción. Revista Iberoamericana de Educación, 1.

Blanco, P. (2012). Paradigma pedagógico ignaciano e investigación - acción. Recuperado el 28 de Enero de 2017, de pedagogiaignaciana.com/ GetFile.ashx?IdDocumento $=1560$

Castillo, L. (2003). Fundamentos y práctica de las competencias ciudadanas. Recuperado el 14 de Enero de 2017, de www.santillana.com.co/ rutamaestra/edicion-10/pdf/6.pdf

Constanza, A., Ruíz, S., \& Guerra, M. (2007). Competencias ciudadanas aplicadas a la educación en Colombia. Educación y desarrollo social, 1(2).

Diario La Opinión. (7 de Junio de 2015). Violencia, imparable en el seno familiar. Recuperado el 24 de Enero de 2017, de http://www.laopinion. com.co/c-cuta/violencia-imparable-en-el-senofamiliar-92806\#ATHS

Feo, R. J. (2010). Orientaciones básicas para el diseño de estrategias didácticas. UPEL. Caracas, Venezuela: Tendencias Pedagógicas.

Fernández, A., \& López, M. d. (Enero-Abril de 2014). Educar para la paz. Necesidad de un cambio epistemológico. Revista de Ciencias Sociales, 21(64), 117-142.

Grande, M. (2010). Convivencia escolar. Un estudio sobre buenas prácticas. Revista de Paz y Conflictos, 154-169.

Gros, B., \& Contreras, D. (Septiembre-Diciembre de 2006). La alfabetización digital y el desarrollo de competencias ciudadanas. Revista Iberoameicana(42), España.

Hernández Sampieri, R., Fernández-Collado, C., \& Baptista, P. (2004). ¿Que caracteristicas posee el enfoque cualitativo? En R. Hernández Sampieri, C. Fernández-Collado, \& P.

Jares, X. (1997). Formación Ética y Ciudadana, Documento de trabajo $n^{o} 4$ Perspectiva transversal: Educación en la paz y los derechos humanos. Buenos Aires: Dirección de Currícula.

Miniesterio de Educación Nacional. (Octubre de 2011). Cartilla 1 Brújula. Programa de Competencias Ciudadanas. Recuperado el 22 de Enero de 2017, de http://www.mineducacion. gov.co/1759/w3-article-235147.html

Ministerio de Educación, Cultura y Deporte de España. (19 de Septiembre de 2013). José Ignacio Wert considera la educación "un pilar fundamental para el progreso de un país". Recuperado el 23 de Enero de 2017, de http://www.mecd.gob.es/prensa-mecd/ actualidad/2013/09/20130919-curso-escolar. html

Mockus, A. (2002). ). Convivencia como armonización de ley, moral y cultura. Perspectivas.

Montoya, L. (2012). La lúdica como estretegia para el desarrollo de las competencias ciudadanas. facultad de ciencias sociales y educación. Caldas-Antioquia: corporación universitaria lasallista.

Muñoz, F. (2001). LA PAZ IMPERFECTA ANTE UN UNIVERSO EN CONFLICTO. Granada, España: Universidad de Granada.

Perez, V. (2001). Convivencia escolar: problemas y soluciones. (U. C. Madrid, Ed.) Revista Complutense de Educación, 12(1), 295.

Restrepo, J. C. (2006). Estándares básicos en competencias ciudadanas: una aproximación al problema de la formación ciudadana en colombia. (p. U. Javeriana, ed.) Redalyc, 11(1), 135-175.

Rovere, M. (2012). ¿Qué es una estrategia? 
Ruiz, R. (2006). La convivencia: un modelo de prevención de la violencia. In La convivencia en las aulas: problemas y soluciones. Ministerio de Educación y Ciencia.

Sarle, P. (2006). Juego y aprendizaje escolar: los rasgos del juego en la educación infantil. Buenos Aires, Argentina: Paidos.

Tesouro, M., Ribot, D., Labian, Í., Guillamed, E., \& Aguilera, A. (25 de Febrero de 2007). Mejoremos los procesos de enseñanzaaprendizaje mediante la investigación-acción. Recuperado el 29 de Enero de 2017, de rieoei. org/deloslectores/1624Tesouro.pdf

Van Dijk. (1998). La pragmática de la comunicación literaria. Madrid: Literaria. Madrid: Arco. 